\title{
Study on Capacity Building Evaluation for Heterogeneous Network
}

\author{
Yunusa M. A ${ }^{1}$, Ladan $\mathbf{M}^{2}$, G.N Jola ${ }^{3}$, Agabi P. $\mathbf{I}^{4}$, and Mahdi A. $\mathrm{H}^{5}$ \\ Research Scholar ${ }^{1-5}$, Professor \\ Department of Electrical/Electronic Engineering Technology \\ The Federal Polytechnic Bauchi, State \\ Nigeria
}

\begin{abstract}
There's a rise in hefty data practice by indoor users as a result of using their smartphones laptops routers and much more datarelated as they interact with downloaded imagines videos and other multimedia accessories. This heavy rise in data use can be solved by a heterogeneous network when a cellular network is incorporated with Pico cells into the existing Macro cells station. This will not only improve the spectral efficiency of users per unit area but also help operators to upgrade their existing networks to meet up the demands of users. This paper presents a comparison solution considered for the existing layout and the challenges faced by base station layout design are presented.
\end{abstract}

Key Words: Heterogeneous network, Base station, Power Consumption, Mobile Cellular Network.

\section{INTRODUCTION}

Demand by cell phone users is on the increase as most developing countries now own more than one cell phone this is mainly due to poor quality of service [1]. This evolution in mobile data traffic upsets not only the capacity necessities of the mobile networks, but even the projected quality of service [2]. As a result, it's becoming unbearable for indoor cell phone users who account for the larger population of benefaction compared to outdoor users, especially in developing nations especially Africa. With the current progression in the use of mobile technology applications, there is an increase demand in the use of voice and data services which has created an unparalleled increase for alternative solutions to mobile broadband services [3]. Also it has been observed that there's a numerical increase in the use of mobile devices for medical applications, live broadcast services the internet browsing and many video streaming services and new application for such use are rising every day on the increase as a result higher demand for data which affects data traffic [4].

One of the most noteworthy factor tangled here is that the service providers have been trying to provide the required rates that their customers need. How to reach a higher data rate with a significant but wider and better coverage has postured a discussion threat in trying to meeting the subscribers' demands [5]. The following term has been used in this work for clarity. Signal to noise ratio SNR this refers to the amount of useful signal in any transmission divided by the interference combined with noise, averaged over the simulation time $t$, is measured in $\mathrm{dB}$. Throughput entails the number of received bits during SLS for time $t$ seconds, whether by a user equipment UE throughput or by a cell cell throughput, is measured in Mbps. Spectral Efficiency this refers to the information rate that can be transmitted over a given bandwidth, is measured in bps/ $\mathrm{Hz}$ [6]

\section{2.. SYSTEM LEVEL SIMULATION METRICS}

In this paper, System level Simulation metrics (SLS) is looked-for to gage the impact of HetNets deployment in macro layer on the network general performance, in case of macro-pico scenario, using some key presentation indicators of those mentioned.

\subsection{Heterogeneous Networks Scenarios}

Giving to the category of LPN (femto cell, pico cell, and micro cell) and arrangement mode (either outdoor or indoor), different HetNets scenarios can be used to study and examine. In this paper, the artificial use of scenario is macro and pico with indoor and outdoor placement of pico cells. For us to achieve the main objective of this exploration, the LTE SLS that has been situated used in this research is centered on Matlab. 
Mobile stations are homogeneously generated across the entire network. A set of dynamic mobile station is selected and evenly distributed mobile station assign to each cell. In this scenario we looked at the macro base station scenario in a specific sector and rise the number of users together with the indoor users [7]. The outdoor users are spread randomly over the hexagonal shape cell and others also at the brink so also the indoor users, then we consider the obtainable data each user can access from the distance of the macro base station [8].

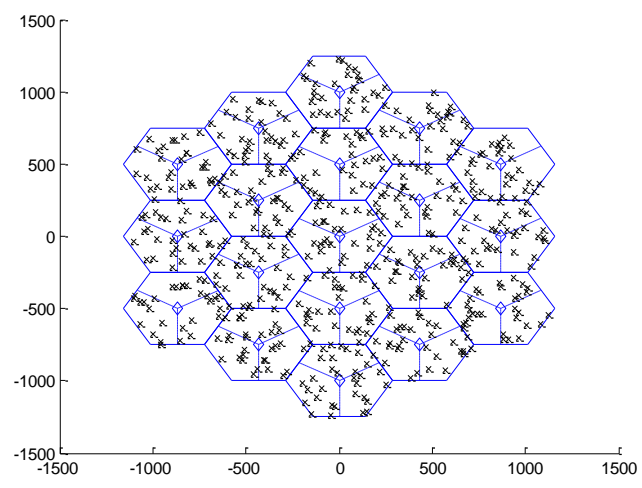

Figure 1 SLS, Network layout for macro layer.

Considering the spectral efficiency of the network Shannon formula was used

\subsection{Shannon's Channel Capacity}

Shannon derived the following capacity formula for an additive white Gaussian noise channel (AWGN) [9]

$\mathrm{C}=\mathrm{W} \log 2(1+\mathrm{S} / \mathrm{N})[\mathrm{bits} / \mathrm{second}]$

- $\quad \mathrm{W}$ is the bandwidth of the channel in $\mathrm{Hz}$

- $\quad \mathrm{S}$ is the signal power in watts

- $\quad \mathrm{N}$ is the total noise power of the channel watts

In statistics, linear regression models often take the form of something like this

Channel Coding Theorem (CCT): The theorem has two parts.

1. Its direct part says that for rate $\mathrm{R}<\mathrm{C}$ there exists a coding system with arbitrarily low block and bit error rates as we let the code length $\mathrm{N} \rightarrow \infty$.

2. The converse part states that for $\mathrm{R} \geq \mathrm{C}$ the bit and block error rates are strictly bounded away from zero for any coding system

The CCT therefore establishes rigid limits on the maximal supportable transmission rate of an additive white Gaussian noise channel (AWGN) channel in terms of power and bandwidth [10].

\subsection{Bandwidth Efficiency}

Bandwidth Efficiency characterizes how efficiently a system uses its allotted bandwidth and is defined as:

$\eta=\underline{\text { Transmission Rate }}[\mathrm{bits} / \mathrm{s} / \mathrm{Hz}]$

Channel Bandwidth W

From it we calculate the Shannon limit as

$$
\eta_{\max }=\log _{2}\left(1+\frac{S}{N}\right)
$$


While Average Signal Power S can be expressed as:

$$
S=\frac{k E_{b}}{T}=R E_{b}
$$

- $\quad \mathrm{E}_{\mathrm{b}}$ is the energy per bit

- $\quad \mathrm{k}$ is the number of bits transmitted per symbol

- $\quad \mathrm{T}$ is the duration of a symbol

- $\quad \mathrm{R}=\mathrm{k} / \mathrm{T}$ is the transmission rate of the system in bits/s.

- $\quad \mathrm{S} / \mathrm{N}$ is called the signal-to-noise ratio

- $\quad \mathrm{N}=\mathrm{N} 0 \mathrm{~W}$ is the total noise power

- $\quad$ N0 is the one-sided noise power spectral density

Here we obtained the Shannon limit in relatives of the bit energy and noise power spectral density, given by:

$$
\eta=\log _{2}\left(1+\frac{R E_{b}}{N_{0} W}\right)
$$

to obtain the least bit of energy required for consistent transmission, we use the Shannon bound:

$$
\frac{E_{b}}{N_{0}} \geq \frac{2^{\eta_{\max }-1}}{\eta_{\max }}
$$

Fundamental limit: For infinite amounts of bandwidth, i.e., $\eta_{\max } \rightarrow 0$, we obtain

$$
\frac{E_{b}}{N_{0}} \geq \frac{2^{\eta_{\max }-1}}{\eta_{\max }}=\ln (2)=-1.59 \mathrm{~dB}
$$

This is the total minimum signal energy to noise power spectral density ratio required to constantly transmit one bit of information.

\subsection{Normalized Capacity}

for the bandwidth W, normalize formulas per signal dimension [11]. This is useful when the question of waveforms and pulse shaping is not a dominant issue, since it allows one to disregard these considerations by treating signal dimensions.

$C_{d}=\frac{1}{2} \log _{2}\left(1+2 \frac{R E_{b}}{N_{0} W}\right)[$ bits $/$ dimension $]$

$C_{d}=\log _{2}\left(1+\frac{R E_{b}}{N_{0}}\right)[$ bits/complex dimension $]$

Applying similar operations as above, we obtain the Shannon bound stabilized per dimension as

$\frac{E_{b}}{N_{0}} \geq \frac{2^{2 c_{d}}-1}{2 C_{d}} ; \frac{E_{b}}{N_{0}} \geq \frac{2^{C_{C}}-1}{C_{c}}$

\subsection{BLER-SNR}

Block Error Rate BLER are set of adaptive white Gaussian noise (AWGN) link-level performance which are necessary to assess block (BLER) at the receiver for received transport blocks (TBs), since BLER at the receiver defines whether TB was received correctly or otherwise and HARQ ACK/NAK reporting is subsequently produced, and also gives a certain resource allocation and modulation and coding schemes (MCS) [12]. In the LTE SLS, and for ease only a subsection of MCSs is simulated, this subsection includes 15 different MCSs each driven by 15 channel quality indicator (CQI). This subsection of MCSs are been chosen so that there is a unmistakable relationship with CQIs. The defined CQIs use coding rates between 1/13 and 1 combined with 4-QAM, 16-QAM, and 64-QAM modulations. Assessing BLER is achieved by SINR-to-BLER which requires an effective SNR value that is obtained from the set of subcarriers-SINRs assigned to UE TB to an AWGN equivalent SNR. 


\section{MODEL}

\subsection{UE's Deployments}

UEs are released in what has been termed region of interest (ROI), which could be the entire network layout or a part of it. ROI is the area where UEs movement and transmission of DL channel are replicated. In ROI the UEs and eNBs are placed within a certain simulation length and UEs are stirred in each transmission time intervals (TTIs), where TTI is equal to 1 ms. This is determined by a random path for each user and a station with displacement equal to the UE's speed introduced when the channel was produced. The more UE is still around ROI, the UE make sure not to change its direction with each interval step If a UE leaved the specified ROI, it would be rearranged randomly within it while handover a new movement route to this UE, from where movement begins when in use within thenetwork layout both in and outside its layout [13].

\subsection{PICO LAYER}

LPNs deployment (Pico cells) involves a new layer to set in them into the macro layer. The method that has been used to organize outdoor pico cells is showed in figure 1, where one pico cell is modelled as a 2-dimensional rectangular block (100 x $100 \mathrm{meters)}$ and positioned randomly in separate site sector with a least distance between macro cell and pico cell equal to $40 \mathrm{~m}$. Using this method may allow to home two pico cells that belong to adjacent macro cells very close, to evade that a least separation between pico cells which is about $40 \mathrm{~m}$ is applied. To decrease the difficulty and running time of SLS, pico cells are positioned only in the central macro cell and its neighboring surrounding. This the same practice that is used to retain macro cell UEs within the expected network layout.

(ROI) is used for each pico cell, in order to elude passing pico cell UEs pico’s region to macro's region.

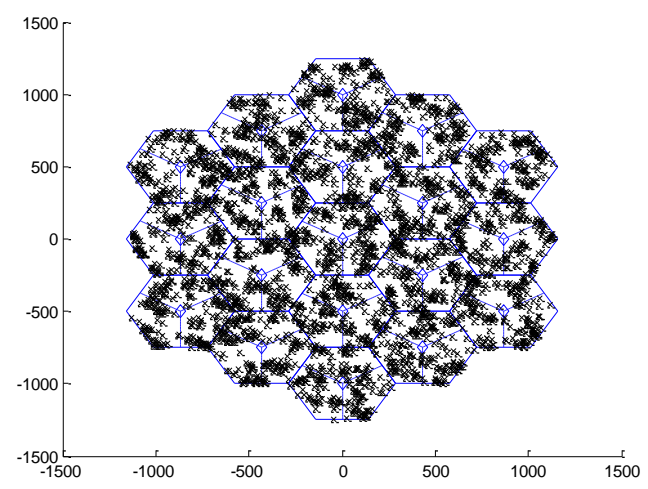

Figure 2: SLS, Outdoor Pico cells deployment within macro layout.

From the way SLS runs we change the spectral efficiency using the software MATLAB code to generate the necessary data each UEs will be able to have the right to use either indoor or outdoor the obtainable throughput required. With the factors we use among others to distribute the transmission blocks to diverse UEs dependent on the SINR.

In this study, several performance metrics were identified and to be used as a means to either evaluate or compare all the studies likewise.

\section{SIMULATION STAGES RESULT AND DISCUTIONS}

In this paper, there are two main phases of simulation that were used to examine how Heterogeneous network could be advance on the cellular network. From the simulation the following results were obtained; 

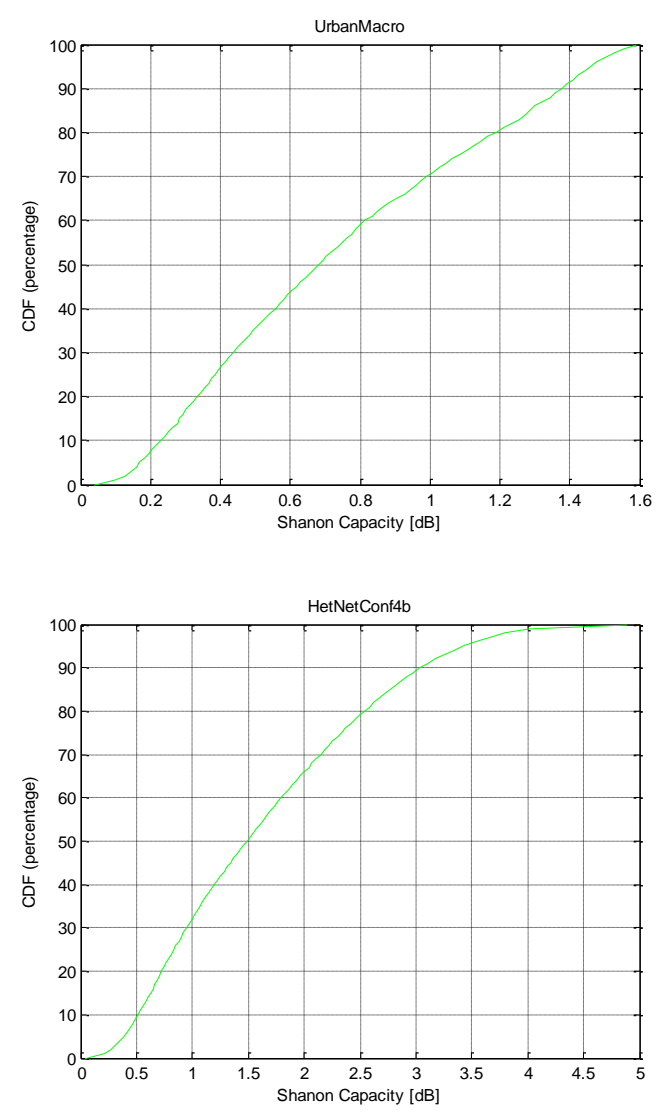

Figure 3: Heterogeneous network, CDF curves of Average UEs SINR (a) macro and (b) with pico cells.

\subsection{SNR}

The SNR values falls slowly away from eNBs and turn out to be less than zero at cell edges. These less than zero values of SNR brings about a severe interference issue for UEs that are positioned at cell-edge. Due to that, a UE has gotten poor services as a result of lower data rates, since the radio channels and particularly the control channels will not work properly under these bad interference conditions. This situation comes to be worse whenever these numerical values go less than zero or more.

Deploying pico cells at cell-edges signifies an efficient solution to handle with this issue as part of the shown pico cell positions within macro layer are based on assumptions. its known that inserting pico cells improves SINR at macro cell-edges. So deploying pico cells at cell-edges has a positive impact on the entire arrangement, since UEs deal with better interference conditions.

Figure 3 illustrates CDFs curves of average UEs SINR for the both scenarios putting all interference factors into consideration. The effects of increasing pico cells are noticeable by comparing the both curves, where the green curve that represents the macro plus pico scenario reflects better interference conditions than macro scenario's curve.

\section{RESULT AND VALIDATION}

Heterogeneous network performance evaluation as earlier mentioned are considered as a significant solution improvement which is introduced and satisfactory to meet and exceed IMT-advanced requirements, as such there can be overall improve the system performance, especially in densely populated areas and cell-edges, where other advanced technologies that has been included in LTE release 8 are incapable. Also as has been observed from the results there is a considerable increase in the spectral efficiency and data rate of a heterogeneous network than the traditional homogeneous network and as such with the placement of pico cells into the existing network there will be considerable rise in user experience with the available network.

\section{CONCLUSIONS}

In this work, the heterogeneous networks have been evaluated as a part of LTE-Advanced features which were implemented to meet IMT-Advanced requirements; these phases have been simulated using system level simulator software making use of a developed edition of the Matlab. The outcomes that have been obtained using the simulator in each step have been used to evaluate this study their benefits. 
The conclusions from this analysis follows that Heterogeneous deployment increases the system overall performance when compared to the standard cellular networks with improvement in both capacity and coverage.

\section{REFERENCES}

[1] Bhosale, D. V, \& Jadhav, P. V. D. (2015). Review : eICIC in Long-Term Evolution HetNets, 5(1), $900-904$.

[2] Ahmad, M. B. (2013). Macro and Femto Network Aspects for Realistic LTE Usage Scenarios with Interference Management.

[3] Nuri, O., Yilmaz, C., \& Networks, N. S. (2010). Self-Optimization of Coverage and Capacity in LTE using Adaptive Antenna Systems, (February).

[4] Ali, N. A., Ouda, M., Hassanein, H., \& Kubbar, O. (2015). Resource allocation with interference mitigation in femtocellular networks Resource allocation with interference mitigation, (July). https://doi.org/10.1002/wcm.2339

[5] Audu, W. M., Onwuka, E. N., Ugweje, O., \& Aibinu, M. A. (2012). Femtocell Technology: A Viable Indoor Coverage Solution

[6] Ahmad, M. B. (2013). Macro and Femto Network Aspects for Realistic LTE Usage Scenarios with Interference Management

[7] Farroha, B. S. (2019). Analyzing the Architecture Advantages and Vulnerabilities in Heterogeneous 5G Wireless Networks. 2019 IEEE International Systems Conference (SysCon), 1-8.

[8] Maurya, S., Jain, V. K., \& Chowdhury, D. R. (2019). Journal of Network and Computer Applications Delay aware energy efficient reliable routing for data transmission in heterogeneous mobile sink wireless sensor network. Journal of Network and Computer Applications, 144(April), 118-137. https://doi.org/10.1016/j.jnca.2019.06.012

[9] Joud, M. A. (2013). Pico Cell Range Expansion toward LTE-Advanced

[10] Deebak, B. D. (2018). Analyzing enhanced real-time uplink scheduling algorithm in 3GPP LTE-advanced networks using multimedia systems, (January), 1-22. https://doi.org/10.1002/ett.3443

[11] Maheshwari, S., \& Netalkar, P. (2020). DISCO: Distributed Control Plane Architecture for Resource Sharing in Heterogeneous Mobile Edge Cloud Scenarios, (July)

[12] Saha, R. K. (2019). Multi-band Spectrum Sharing with Indoor Small Cells in Hybrid Satellite-Mobile Systems. 2019 IEEE 90th Vehicular Technology Conference (VTC2019-Fall), (1), 1-7.

[13] Zhang, X., Gu, W., Zhang, H., \& Wang, M. (2019). 5G / B5G WIRELESS SYSTEMS Hybrid Communication Path Orchestration for 5G Heterogeneous Ultra-Dense Networks, (August), 112-118. 